\title{
Exciting news from the adult mouse subventricular zone
}

\section{Mila Komitova and Flora M. Vaccarino*}

Yale University School of Medicine, New Haven, CT, USA

*Correspondence: flora.vaccarino@yale.edu

\section{A commentary on}

Adult generation of glutamatergic olfactory bulb interneurons.

by M. S. Brill, J. Ninkovic, E. Winpenny, R. D. Hodge, I. Ozen, R. Yang, A. Lepier, S. Gascon, F. Erdelyi, G. Szabo, C. Parras, F. Guillemot, M. Frotscher, B. Berninger, R. F. Hevner, O. Raineteau and M. Gotz (2009). Nat. Neurosci. 12, 1524-1533.

In the adult mammalian brain the forebrain subventricular zone (SVZ) is a source of olfactory bulb (OB) GABAergic neurons. It is by now well established that astrocytelike cells via fast-amplifying progenitor cells generate neuronal precursors that mature into functional neurons (Kriegstein and Alvarez-Buylla, 2009). The potential of the adult SVZ to generate neuronal progenitors that are recruited to lesioned brain regions in various injury models, has opened up new vistas in attempting to enhance neurologic recovery through neuronal replacement (Lindvall and Kokaia, 2006; Zhang et al., 2008).

Previous studies have established that the adult mouse SVZ contains heterogenous progenitor populations based on their embryonic origins and their potential to generate different subtypes of GABAergic interneurons (Merkle et al., 2007; Young et al., 2007). Brill and colleagues add a further level of complexity by demonstrating that some neural progenitors in the adult SVZ generate a subtype of glutamatergic neurons in the OB. The authors examined the transcription factors Neurogenin 2 (Neurog2), Tbr2 and Tbr1, known to be associated with acquisition of glutamatergic neuronal fate during cortical development. Using co-labeling with different transcription factors, 5-bromo-2'-deoxyuridine (BrdU) birth dating and transgenic mice with transcription factor-specific reporter expression, Brill et al. demonstrated lineage progression from $\mathrm{Pax}^{+} / \mathrm{Mash}^{+}$cells, to intermediary progenitor cells expressing Neurog 2 and Tbr2 and ultimately to
Tbr $1^{+}$postmitotic immature neurons. The Neurog $2^{+}$, Tbr $2^{+}$and $\mathrm{Tbr} 1^{+}$cells were only found in the dorsal region of the SVZ and proximal rostral migratory stream (RMS), as opposed to the progenitors of GABAergic interneurons, which were present over the entire SVZ. The authors next used their previously validated GLASTCre::ERT2 mice, in which the promoter of the astrocytespecific glutamate transporter (GLAST) drives tamoxifen-inducible Cre recombination in astroglial cells (Mori et al., 2006). Fate mapping using the GLASTCre::ERT2; R26R-CFP mice demonstrated that the $\mathrm{Tbr} 2^{+}$progenitors observed in the SVZ and RMS originated from $\mathrm{GLAST}^{+}$astrocyte-like cells.

The authors showed that proliferating cells in the adult SVZ generated a subtype of glutamatergic neuron, which based on location and morphology was categorized as a short-axon juxtaglomerular $\mathrm{OB}$ interneuron. Glutamatergic fate of the adult-generated neurons was confirmed by demonstrating co-expression of vesicular glutamate transporter 2 (vGluT2). Only a small fraction of adult-generated $\mathrm{BrdU}^{+}$ cells in the glomerular layer of the $\mathrm{OB}$ were found to co-express vGluT2 (2\%). Brill et al. replicated the in vivo data by in vitro experiments where cultured SVZ cells were found to generate a small fraction of glutamatergic neurons which exhibited functional synaptic transmission. In order to further demonstrate that Neurog $2^{+}$progenitors were indeed the source of the adult-born juxtaglomerular neurons, the authors also analyzed adult-generated Neurog2 lineage cells in the OB of Neurog2::Cre mice carrying a Z/EG reporter. A small fraction of $\mathrm{BrdU}^{+}$adult born cells in the glomerular layer of the $\mathrm{OB}$ were found to originate from the Neurog 2 lineage (5\%). It is unclear what is the number and proportion of Tbrexpressing excitatory neurons with respect to the overall number of adult-generated neurons in the SVZ, as well as what percentage of these cells reaches the OB. However, this may be hard to assess given the small number of $\mathrm{BrdU}^{+}$cells that acquires an excitatory fate. It seems that only a small proportion of the numerous cells that originally expressed Neurog2, Tbr2, Tbr1 in the SVZ reaches the periglomerular regions, raising the question what happens to the remaining cells.

The adult-generated glutamatergic neuronal progenitors down regulated Tbr 2 and even Tbr1 before or just after reaching the OB despite acquiring glutamatergic fate, as assessed by vGluT2 expression. The authors did observe many vGluT ${ }^{+}$cells that co-expressed Tbr1 and Tbr2 in the glomerular layer. However, these glomerular layer $\mathrm{Tbr} 2^{+}$cells were found to be generated embryonically. The significance of the difference in Tbr transcription factor expression between embryonically and adult-generated periglomerular glutamatergic neurons needs to be examined further. It is also possible that BrdU labeling of these cells somehow interferes with their normal transcription factor expression profile. Perhaps long term fate mapping of excitatory OB neurons generated in the adult using GlastCre::ERT2;R26R-CFP mice without using BrdU could answer this question.

Lastly, the authors showed recruitment of newly-generated Tbr2 $2^{+}$neuroblasts from the SVZ toward the lesioned cerebral cortex after targeted callosal projection neuron degeneration. Some of the Tbr2 lineage cells expressed the upper layer identity transcription factor Cuxl. Even though the Brill et al. study does not provide any quantification of the $\mathrm{Tbr} 2^{+}$ neuronal progenitor recruitment, this seems to be a relatively rare phenomenon. The generation of new cortical pyramidal neurons in adulthood in response to apoptosis of resident neurons had already been shown (Magavi et al., 2000), however the source of these new neurons was unclear. The finding that new $\mathrm{Tbr} 1^{+}$neurons can be generated in adulthood has important implications for pathological conditions of the cerebral cortex, as it implies that 
SVZ cells can represent a source of cortical excitatory neurons. The idea that SVZ progenitors can generate pyramidal cortical neurons, as demonstrated by Brill et al., agrees with an earlier study which showed enhanced generation of $\mathrm{Tbr}^{+}$ neurons in the mouse neocortex after chronic postnatal hypoxia, a clinically relevant model for neuropathology in preterm infants (Fagel et al., 2009). Together, these papers suggest that new excitatory neurons can be integrated into the postnatal neocortex. The paper of Brill et al. lays important groundwork for future research avenues leading to an understanding of the molecular mechanisms by which progenitor cells migrate and integrate into the cerebral cortex.

The intriguing findings of Brill et al. emphasize that the plasticity of olfactory circuitry is not confined exclusively to inhibitory neurons. The major limitation of this study is the lack of understanding concerning the physiological role of adultborn olfactory and also cortical neurons for brain function. Despite this limitation, the study of Brill et al. is the first to extend the cellular repertoire of the SVZ to excitatory neuron progenitors, which was previously thought to occur only in embryogenesis. The maintenance of this large variety of cellular precursors in the adult SVZ niche raises our hope that the balanced replacement of different neuronal subtypes can be achieved in various lesion models, and that significant improvement of function in neurological or neuropsychiatric disorders can be attained.

\section{REFERENCES}

Fagel, D. M., Ganat, Y., Cheng, E., Silbereis, J., Ohkubo, Y., Ment, L. R., and Vaccarino, F. M. (2009). Fgfr 1 is required for cortical regeneration and repair after perinatal hypoxia. J. Neurosci. 29, 1202-1211. doi: 29/4/1202 [pii] 10.1523/ JNEUROSCI.4516-08.2009.

Kriegstein, A., and Alvarez-Buylla, A. (2009). The glial nature of embryonic and adult neural stem cells. Annu. Rev. Neurosci. 32, 149-184. doi: 10.1146/ annurev.neuro.051508.135600.

Lindvall, O., and Kokaia, Z. (2006). Stem cells for the treatment of neurological disorders. Nature 441, 1094-1096. doi: nature04960 [pii] 10.1038/ nature 04960 .

Magavi, S. S., Leavitt, B. R., and Macklis, J. D. (2000). Induction of neurogenesis in the neocortex of adult mice. Nature 405, 951-955.
Merkle,F. T., Mirzadeh,Z., and Alvarez-Buylla, A. (2007). Mosaic organization of neural stem cells in the adult brain. Science 317, 381-384. doi: 1144914 [pii] 10.1126/science.1144914.

Mori, T., Tanaka, K., Buffo, A., Wurst, W., Kuhn, R., and Gotz, M. (2006). Inducible gene deletion in astroglia and radial glia - a valuable tool for functional and lineage analysis. Glia 54, 21-34.

Young, K. M., Fogarty, M., Kessaris, N., and Richardson, W. D. (2007). Subventricular zone stem cells are heterogeneous with respect to their embryonic origins and neurogenic fates in the adult olfactory bulb. J. Neurosci, 27, 8286-8296. doi: 27/31/8286 [pii] 10.1523/JNEUROSCI.0476-07.2007.

Zhang, R. L., Zhang, Z. G., and Chopp, M. (2008) Ischemic stroke and neurogenesis in the subventricular zone. Neuropharmacology 55, 345-352. doi: S0028-3908(08)00159-7 [pii] 10.1016/j.neuropharm.2008.05.027.

Received: 21 December 2009; published online: 27 January 2010.

Citation: Komitova M and Vaccarino FM (2010) Exciting news from the adult mouse subventricular zone. Front. Neurosci. 4:23. doi: 10.3389/neuro.22.001.2010

This article was submitted to Frontiers in Neurogenesis, a specialty of Frontiers in Neuroscience.

Copyright () 2010 Komitova and Vaccarino. This is an open-access article subject to an exclusive license agreement between the authors and the Frontiers Research Foundation, which permits unrestricted use, distribution, and reproduction in any medium, provided the original authors and source are credited. 